PROCEEDINGS OF THE AMERICAN MATHEMATICAL SOCIETY

Volume 124, Number 2, February 1996

\title{
A NOTE ON GENERATORS OF LEAST DEGREE IN GORENSTEIN IDEALS
}

\author{
MATTHEW MILLER AND RAFAEL H. VILLARREAL
}

(Communicated by Wolmer V. Vasconcelos)

\begin{abstract}
Assume $R$ is a polynomial ring over a field and $I$ is a homogeneous Gorenstein ideal of codimension $g \geq 3$ and initial degree $p \geq 2$. We prove that the number of minimal generators $\nu\left(I_{p}\right)$ of $I$ that are of degree $p$ is bounded above by $\nu_{0}=\left(\begin{array}{c}p+g-1 \\ g-1\end{array}\right)-\left(\begin{array}{c}p+g-3 \\ g-1\end{array}\right)$, which is the number of minimal generators of the defining ideal of the extremal Gorenstein algebra of codimension $g$ and initial degree $p$. Further, $I$ is itself extremal if $\nu\left(I_{p}\right)=\nu_{0}$.
\end{abstract}

\section{INTRODUCTION}

Assume $R$ is a polynomial ring over a field and $I$ is a homogeneous Gorenstein ideal of codimension $g \geq 3$ and initial degree $p \geq 2$. Let

$$
e(g, p)=\left(\begin{array}{c}
g+p-1 \\
g
\end{array}\right)+\left(\begin{array}{c}
g+p-2 \\
g
\end{array}\right) \quad \text { and } \quad \nu_{0}=\left(\begin{array}{c}
p+g-1 \\
g-1
\end{array}\right)-\left(\begin{array}{c}
p+g-3 \\
g-1
\end{array}\right) .
$$

A consequence of the Macaulay-Stanley characterization [10] of the Hilbert function of $R / I$ is that the multiplicity of $R / I$ satisfies $e(R / I) \geq e(g, p)$. Given codimension $g$ and initial degree $p$, a graded Gorenstein algebra $R / J$ with multiplicity $e(R / J)=e(g, p)$ is extremal in the sense of Schenzel [8]. This has strong structural implications: the minimal free resolution of $R / J$ must be pure and almost linear; consequently all the betti numbers $\beta_{i}(R / J)$ are determined, and in particular $J$ is generated by $\nu_{0}$ forms of degree $p$.

Conjecture 1.1. If $I$ is a graded Gorenstein ideal of height $g \geq 3$ and initial degree $p \geq 2$, then $\nu\left(I_{p}\right) \leq \nu_{0}$. If, further, $\nu\left(I_{p}\right)=\nu_{0}$, then $I$ is extremal, or equivalently, $e(R / I)=e(g, p)$. Consequently if $\nu\left(I_{p}\right)=\nu_{0}$, then $I=\left(I_{p}\right)$.

Results in the literature dealing with Cohen-Macaulay ideals (such as $[3,4,7]$ ) give upper bounds for $\nu(I)$ in terms of codimension, initial degree, and multiplicity $e(R / I)$. Our intent is to elucidate the multiplicity information that is already determined by the codimension and initial degree. In general one expects no such conclusion, but for graded Gorenstein algebras the symmetry of the $h$-vector $H(R / I)$ can be effectively exploited. Naturally, therefore, one would not expect any such results to generalize to the non-graded case.

Received by the editors June 6, 1994 and, in revised form, August 25, 1994.

1991 Mathematics Subject Classification. Primary 13H10; Secondary 13D40.

The first author was supported by the National Science Foundation.

The second author was partially supported by COFAA-IPN, CONACyT and SNI, México.

(c)1996 American Mathematical Society 
One might hope for even stronger estimates than suggested by Conjecture 1.1, for instance that $\nu(I) \leq \nu_{0}$ or even $\beta_{i}(R / I) \leq \beta_{i}(R / J)$ for all $i$. We present a counterexample, but we suspect there are reasonable conditions under which the stronger estimates might hold.

In Remark 2.1 we point out one further elaboration of the Conjecture, namely that it is not the case that every value in the range $\left[g, \nu_{0}\right]$ is exhibited by $\nu\left(I_{p}\right)$ for some $I$. There are obstructions to certain values, which can be proved by using the same ideas that establish the main inequality.

\section{THE MAIN THEOREM}

Theorem 2.1. If $I$ is a graded Gorenstein ideal of codimension $g \geq 3$ and initial degree $p \geq 2$, then

$$
\nu\left(I_{p}\right) \leq \nu_{0}=\left(\begin{array}{c}
p+g-1 \\
g-1
\end{array}\right)-\left(\begin{array}{c}
p+g-3 \\
g-1
\end{array}\right)
$$

and $I$ is itself extremal if equality holds.

Proof. If either $\nu\left(I_{p}\right)>\nu_{0}$, or $\nu\left(I_{p}\right)=\nu_{0}$ and $I$ is not extremal, then by the symmetry of the $h$-vector $H(R / I)$ there is some $j \geq p$ so that

$$
H(R / I)=\left(h_{0}, h_{1}, \ldots, h_{p-1}, h_{p}, \ldots, h_{j}, h_{p-1}, \ldots, h_{1}, h_{0}\right),
$$

where $h_{j}=h_{p} \leq\left(\begin{array}{c}p+g-3 \\ g-1\end{array}\right)=h_{p-2}$. The idea of the argument is to use the Macaulay bound $h_{j}^{\langle j\rangle}$ (see $[1,3,6,10]$ ) for $h_{j+1}$ to see that such a small value of $h_{j}$ can not grow to such a large value of $h_{j+1}=h_{p-1}=\left(\begin{array}{c}p+g-2 \\ g-1\end{array}\right)$. We recall that this estimate is calculated from the binomial expansion for $h_{j}$ :

$$
h_{j}=\left(\begin{array}{c}
a_{j} \\
j
\end{array}\right)+\left(\begin{array}{c}
a_{j-1} \\
j-1
\end{array}\right)+\cdots+\left(\begin{array}{c}
a_{i} \\
i
\end{array}\right)
$$

where $a_{j}>a_{j-1}>\cdots>a_{i} \geq i \geq 1$. Then

$$
h_{j+1} \leq h_{j}^{\langle j\rangle}=\left(\begin{array}{c}
a_{j}+1 \\
j+1
\end{array}\right)+\left(\begin{array}{c}
a_{j-1}+1 \\
j
\end{array}\right)+\cdots+\left(\begin{array}{c}
a_{i}+1 \\
i+1
\end{array}\right) .
$$

We may assume that $h_{j}>j$, for if not, then $h_{j} \leq j$ would imply that $a_{\ell}=\ell$ for all $\ell$, and hence $h_{j} \geq h_{j+1}$, which contradicts our assumption. Notice that by grouping the terms of (1) according to the value of $a_{\ell}-\ell$ the binomial expansion for $h_{j}$ can be written as

$$
\begin{aligned}
h_{j} & =\sum_{n=0}^{r}\left[\left(\begin{array}{c}
j_{n}+k_{n} \\
j_{n}
\end{array}\right)+\left(\begin{array}{c}
j_{n}-1+k_{n} \\
j_{n}-1
\end{array}\right)+\cdots+\left(\begin{array}{c}
j_{n}-i_{n}+k_{n} \\
j_{n}-i_{n}
\end{array}\right)\right] \\
& =\sum_{n=0}^{r}\left[\left(\begin{array}{c}
j_{n}+k_{n}+1 \\
j_{n}
\end{array}\right)-\left(\begin{array}{c}
j_{n}-i_{n}+k_{n} \\
j_{n}-i_{n}-1
\end{array}\right)\right],
\end{aligned}
$$

where $a_{j}-j=k_{0}>k_{1}>\cdots>k_{r} \geq 0, j=j_{0}>j_{1}>\cdots>j_{r}, j_{r}-i_{r}=i$, and $j_{n}=j_{n-1}-i_{n-1}-1$ for $1 \leq n \leq r$. Set $k=k_{0}$. Since $p \leq j$ and $\left(\begin{array}{c}j+k \\ j\end{array}\right) \leq h_{j} \leq$ $\left(\begin{array}{c}p+g-3 \\ g-1\end{array}\right)$ it follows that $k \leq g-2$. From (1), (2), and (3), together with Pascal's 
identity and $\left(\begin{array}{c}a+b+1 \\ b+1\end{array}\right)=\frac{a+1}{b+1}\left(\begin{array}{c}a+b+1 \\ b\end{array}\right)$, we have

$$
\begin{aligned}
h_{j+1}-h_{j} & \leq \sum_{n=0}^{r}\left[\left(\begin{array}{c}
j_{n}+k_{n}+1 \\
j_{n}+1
\end{array}\right)-\left(\begin{array}{c}
j_{n}-i_{n}+k_{n} \\
j_{n}-i_{n}
\end{array}\right)\right] \\
& =\sum_{n=0}^{r}\left[\frac{k_{n}+1}{j_{n}+1}\left(\begin{array}{c}
j_{n}+k_{n}+1 \\
j_{n}
\end{array}\right)-\frac{k_{n}+1}{j_{n}-i_{n}}\left(\begin{array}{c}
j_{n}-i_{n}+k_{n} \\
j_{n}-i_{n}-1
\end{array}\right)\right] .
\end{aligned}
$$

On the other hand from the upper bound on $h_{j}$ and $h_{j+1}=h_{p-1}$ we get

$$
\frac{g-1}{p-1} h_{j} \leq\left(\begin{array}{c}
p+g-3 \\
g-2
\end{array}\right) \leq h_{j+1}-h_{j}
$$

Since $(g-1) /(p-1)>(k+1) /(j+1)$ it follows from $(3)$ and the last two inequalities that $F_{0}<0$, where for $0 \leq s \leq r$ we set

$$
\begin{aligned}
F_{s}=\sum_{n=s}^{r} & {\left[\left(\frac{k_{s}+1}{j_{s}+1}-\frac{k_{n}+1}{j_{n}+1}\right)\left(\begin{array}{c}
j_{n}+k_{n}+1 \\
j_{n}
\end{array}\right)\right] } \\
& +\sum_{n=s}^{r}\left[\left(\frac{k_{n}+1}{j_{n}-i_{n}}-\frac{k_{s}+1}{j_{s}+1}\right)\left(\begin{array}{c}
j_{n}-i_{n}+k_{n} \\
j_{n}-i_{n}-1
\end{array}\right)\right] .
\end{aligned}
$$

To derive a contradiction we are going to show the inequalities

$$
F_{0}>F_{1}>\cdots>F_{r}=\left(\frac{k_{r}+1}{j_{r}-i_{r}}-\frac{k_{r}+1}{j_{r}+1}\right)\left(\begin{array}{c}
a_{i} \\
i-1
\end{array}\right)>0
$$

Assume $1 \leq s+1 \leq r$. Notice that

$$
\begin{aligned}
0 & \leq \sum_{n=s}^{r-1}\left[\left(\begin{array}{c}
j_{n}-i_{n}+k_{n} \\
j_{n}-i_{n}-1
\end{array}\right)-\left(\begin{array}{c}
j_{n+1}+k_{n+1}+1 \\
j_{n+1}
\end{array}\right)\right]+\left(\begin{array}{c}
j_{r}-i_{r}+k_{r} \\
j_{r}-i_{r}-1
\end{array}\right) \\
& =\left(\begin{array}{c}
j_{s}-i_{s}+k_{s} \\
j_{s}-i_{s}-1
\end{array}\right)-\sum_{n=s+1}^{r}\left[\left(\begin{array}{c}
j_{n}+k_{n}+1 \\
j_{n}
\end{array}\right)-\left(\begin{array}{c}
j_{n}-i_{n}+k_{n} \\
j_{n}-i_{n}-1
\end{array}\right)\right] .
\end{aligned}
$$

Therefore

$$
\left(\begin{array}{c}
j_{s}-i_{s}+k_{s} \\
j_{s}-i_{s}-1
\end{array}\right) \geq \sum_{n=s+1}^{r}\left(\begin{array}{c}
j_{n}+k_{n}+1 \\
j_{n}
\end{array}\right)-\sum_{n=s+1}^{r}\left(\begin{array}{c}
j_{n}-i_{n}+k_{n} \\
j_{n}-i_{n}-1
\end{array}\right) .
$$

Let $A_{s}$ denote the first summation and $B_{s}$ the second in this last inequality; clearly $A_{s}-B_{s}>0$. Also note that

$$
\frac{k_{s}+1}{j_{s}-i_{s}}>\frac{k_{s}+1}{j_{s}+1} \quad \text { and } \quad \frac{k_{s}+1}{j_{s}-i_{s}}>\frac{k_{s+1}+1}{j_{s+1}+1} .
$$


Putting these together, we compute

$$
\begin{aligned}
F_{s}-F_{s+1}= & \left(\frac{k_{s}+1}{j_{s}-i_{s}}-\frac{k_{s}+1}{j_{s}+1}\right)\left(\begin{array}{c}
j_{s}-i_{s}+k_{s} \\
j_{s}-i_{s}-1
\end{array}\right) \\
& \quad+\left(\frac{k_{s}+1}{j_{s}+1}-\frac{k_{s+1}+1}{j_{s+1}+1}\right) A_{s}+\left(\frac{k_{s+1}+1}{j_{s+1}+1}-\frac{k_{s}+1}{j_{s}+1}\right) B_{s} \\
\geq & \left(\frac{k_{s}+1}{j_{s}-i_{s}}-\frac{k_{s}+1}{j_{s}+1}\right)\left(A_{s}-B_{s}\right)+\left(\frac{k_{s}+1}{j_{s}+1}-\frac{k_{s+1}+1}{j_{s+1}+1}\right) A_{s} \\
\quad+\left(\frac{k_{s+1}+1}{j_{s+1}+1}-\frac{k_{s}+1}{j_{s}+1}\right) B_{s} & \left(\frac{k_{s}+1}{j_{s}-i_{s}}-\frac{k_{s+1}+1}{j_{s+1}+1}\right)\left(A_{s}-B_{s}\right)>0 .
\end{aligned}
$$

Hence $F_{0}>F_{r}>0$, which contradicts the earlier observation that $F_{0}<0$.

Remark 2.1. We have not worked out in general which values of $\nu\left(I_{p}\right)<\nu_{0}$ are forbidden, but point out that the symmetry of $H(R / I)$ restricts the possibilities of small values of $\nu\left(I_{p}\right)$ just as it rules out large values. For example, if $g=4$, then $\nu_{0}=(p+1)^{2}$. Let us see what happens in case $p=4$ and $\nu\left(I_{4}\right)<25$. There is no apparent Hilbert function obstruction to values $\nu\left(I_{4}\right) \leq 15$, but in the range $15<\nu\left(I_{4}\right)<25$ one will have $10<h_{j}=h_{4}<20$ growing to $h_{j+1}=20$, and this suggests that arguments along the lines we have been giving will still be effective. Since $a_{j} \geq j+2$ (notation of equation (1)) is only possible if $j=4$, and $h_{4}=\left(\begin{array}{l}6 \\ 4\end{array}\right)=15$ can grow to $h_{5}=20<\left(\begin{array}{l}7 \\ 5\end{array}\right)$, such arguments can only succeed for $11 \leq h_{j} \leq 14$. In this case we are looking for a gain $h_{j+1}-h_{j} \geq 6$, so the expansion of $h_{j}$ will have to have at least six terms of form $\left(\begin{array}{c}i+1 \\ i\end{array}\right)$; this is clearly impossible for $h_{j} \leq 26$. We conclude that the values $21 \leq \nu\left(I_{4}\right) \leq 24$ do not occur. If $16 \leq \nu\left(I_{4}\right) \leq 20$, then $H(R / I)$ fails to be unimodal. There are no known examples of such sequences for $g=4$ at all, and there is some evidence that they may not be possible. One can interpret our arguments as ruling out "extreme" failure of unimodality, leaving a grey zone of "mild" failure of unimodality open for more investigation.

Remark 2.2. It has been suggested to us that there is the possibility of a very short and elegant argument, at least for the main result that $\nu\left(I_{p}\right) \leq \nu_{0}$, based on the behavior of the combinatorial functions $f(x)=x^{\langle n\rangle}$ and $g(x)=h^{\langle x\rangle}$; see the notation of (2). The key to this argument rests on the observation (for which we do not know a proof) that $g$ is non-increasing.

The next example shows that Theorem 2.1 can not be extended to bound the number of generators in all degrees.

Example 2.1. Let $I=\left(\left(x_{1}^{2}, x_{2}^{4}, x_{3}^{3}, x_{4}^{4}\right):\left(x_{1} x_{2}-x_{3} x_{4}\right)\right)$. One has

$$
\begin{aligned}
& I=\left(x_{1}^{2}, x_{1} x_{2} x_{3}+x_{3}^{2} x_{4}, x_{3}^{3},\right. \\
& \left.\quad x_{1} x_{3}^{2}, x_{2}^{4}, x_{4}^{4}, x_{1} x_{4}^{3}, x_{1} x_{2} x_{4}^{2}+x_{3} x_{4}^{3}, x_{2}^{3} x_{3}^{2}, x_{2}^{3} x_{4}^{3}\right) .
\end{aligned}
$$

Then $R / I$ is a Gorenstein artin algebra with $h$-vector $(1,4,9,13,13,9,4,1)$ and betti sequence $(1,10,18,10,1)$, whereas the $h$-vector for an extremal Gorenstein algebra of codimension four and initial degree two is $(1,4,1)$ and the betti sequence is $(1,9,16,9,1)$; notice that the multiplicity $e(R / I)=54$ is far greater than the minimal value of six exhibited by an extremal algebra. The graded structure of 
the minimal free resolution (especially that the last term is $R(-11)$, whereas in the extremal case it is $R(-6)$ ) suggests that a suitable a priori upper bound on the regularity index of $R / I$, or equivalently on the socle degree, might enable one to bound $\nu(I)$ by $\nu_{0}$.

Remark 2.3. A bound on the regularity index amounts to a hypothesis that the resolution is not too far from being pure. It seems likely that if $R / I$ has a pure minimal free resolution, then $\beta_{i}(R / I) \leq \beta_{i}(R / J)$ for all $i$, where $J$ is an extremal Gorenstein algebra of the same codimension and initial degree as $I$. Purity may well be a stronger hypothesis than one needs; for instance, if $I$ is generated only in degree $p, g=4$, and $R / J$ is an extremal algebra of the same codimension and initial degree, then the theorem shows that $\beta_{1}(R / I) \leq \beta_{1}(R / J)$; symmetry of the minimal free resolution and $\sum_{i=0}^{4}(-1)^{i} \beta_{i}(R / I)=0$ then imply that $\beta_{i}(R / I) \leq \beta_{i}(R / J)$ for all $i$. Mats Boij has shown us numerical computations that suggest the betti numbers of compressed graded Gorenstein algebras, whose resolutions consist of just two almost linear strands, also appear to satisfy this inequality.

The same bound holds in codimension three, without any purity assumption, thanks to the Buchsbaum-Eisenbud structure theorem. For initial degree $p$, the extremal Gorenstein algebra of codimension three has $\beta_{1}=\beta_{2}=2 p+1$.

Theorem 2.2. Let $R$ be a polynomial ring over a field and $I$ be a homogeneous Gorenstein ideal of height three. If $p$ is the initial degree of $I$, then $\nu(I) \leq 2 p+1$ and $\beta_{2}(R / I) \leq 2 p+1$.

Proof. (Sketch) We may assume, without loss of generality, that $R$ is equal to $k\left[x_{1}, x_{2}, x_{3}\right]$ and $A=R / I$ is artinian and local with $\operatorname{socle}(A)=A_{\sigma}$. Then by [2] the minimal free resolution of $A$ has the form

$$
0 \rightarrow R(-\sigma-3) \rightarrow \bigoplus_{j=1}^{\nu} R\left(-n_{j}\right) \stackrel{Y}{\longrightarrow} \bigoplus_{i=1}^{\nu} R\left(-m_{i}\right) \rightarrow R
$$

where $Y$ is an alternating matrix, and the generators $f_{1}, \ldots, f_{\nu}$ of $I$ are the maximal pfaffians of $Y$. If the theorem fails every generator has degree at least $(2 p+1) / 2>p$, which is a contradiction since at least one minimal generator has degree $p$.

\section{ACKNOWLEDGMENT}

Investigation of this problem first began when the first author was on sabbatical at Rutgers University, and continued during a visit to the Instituto Politécnico Nacional; he would like to thank both institutions for their support and hospitality.

\section{REFERENCES}

1. W. Bruns and J. Herzog, Cohen-Macaulay rings, Cambridge University Press, Cambridge, 1993. CMP 94:05

2. D. Buchsbaum and D. Eisenbud, Algebra structures for finite free resolutions, and some structure theorems for ideals of codimension 3, Amer. J. Math. 99 (1977), 447-485. MR 56:11983

3. J. Elias, Three results on the number of generators of ideals, Comm. Algebra 19 (1991), 1387-1408. MR 92i:13010

4. J. Elias, L. Robbiano, and G. Valla, Numbers of generators of perfect ideals, Nagoya Math. J. 123 (1991), 39-76. MR 92h:13023

5. R. Fröberg and D. Laksov, Compressed algebras, Complete intersections (S. Greco and R. Strano, eds.), Lecture Notes in Math., vol. 1092, Springer-Verlag, Heidelberg, 1984, pp. 121151. MR 86f: 13012 
6. L. Robbiano, Introduction to the theory of Hilbert functions, Queen's Papers in Pure and Applied Math., vol. 85, Queen's Univ., Kingston, ON, 1991, pp. B1-B26. MR 92g:13019

7. M. E. Rossi and G. Valla, Multiplicity and t-isomultiple ideals, Nagoya Math. J. 110 (1988), 81-111. MR 89g: 13011

8. P. Schenzel, Über die freien Auflösungen extremaler Cohen-Macaulay-Ringe, J. Algebra 64 (1980), 93-101. MR 81j:13024

9. R. Stanley, Hilbert functions of graded algebras, Adv. Math. 28 (1978), 57-83. MR 58:5637

10. - Combinatorics and commutative algebra, Birkhäuser, Boston, 1983. MR 85b:05002

Department of Mathematics, University of South Carolina, Columbia, South CarOLINA 29208

E-mail address: miller@math.sc.edu

Departamento de Matemáticas, Escuela Superior de Física y Matemáticas, Instituto Politécnico Nacional, Unidad Adolfo López Mateos, México, D.F. 07300

E-mail address: vila@esfm.ipn.mx 